\title{
Potassium Acetate
}

National Cancer Institute

\section{Source}

National Cancer Institute. Potassium Acetate. NCI Thesaurus. Code C47677.

The acetate salt form of potassium, an essential macromineral. Potassium maintains intracellular tonicity, is required for nerve conduction, cardiac, skeletal and smooth muscle contraction, production of energy, the synthesis of nucleic acids, maintenance of blood pressure and normal renal function. This agent has potential antihypertensive effects and when taken as a nutritional supplement may prevent hypokalemia. 